Revista Brasileira de Agricultura Irrigada v.11, nº.6, p. 1862 - 1875, 2017

ISSN 1982-7679 (On-line)

Fortaleza, CE, INOVAGRI - http://www.inovagri.org.br

DOI: $10.7127 /$ rbai.v11n600642

Protocolo 642.17 - 21/03/2017 Aprovado em 07/04/2017

\title{
REPOSIÇÃO HÍDRICA E ADUBAÇÃO NITROGENADA NA CANA-DE-AÇÚCAR VIA GOTEJAMENTO SUBSUPERFICIAL: CANA-PLANTA E CANA-SOCA
}

\author{
Nelmicio Furtado da Silva ${ }^{1}$, Fernando Nobre Cunha ${ }^{2}$, Marconi Batista Teixeira ${ }^{3}$, Frederico \\ Antonio Loureiro Soares ${ }^{4}$, Vitor Marques Vidal ${ }^{5}$, Wilker Alves Morais ${ }^{6}$
}

\section{RESUMO}

A melhoria da qualidade da cana-de-açúcar pode ser obtida com o uso da irrigação, que além de aumentar a produtividade da cana-de-açúcar, promove melhorias nos índices de qualidade da matéria-prima, porém vários fatores podem interferir na qualidade final, portanto a cana-deaçúcar irrigada depende da quantidade de água aplicada, do manejo de irrigação combinado com a quantidade certa de adubação. Partindo da hipótese de que existe interação entre reposição hídrica e adubação nitrogenada nos diferentes ciclos de cultivo, objetivou-se com este trabalho acompanhar nas diferentes fases de crescimento e desenvolvimento da cana-de-açúcar, submetida a diferentes reposições hídricas e adubação nitrogenada via gotejamento subsuperficial nas condições de cana-planta e soca. O delineamento experimental utilizado foi o de blocos ao acaso, analisado em esquema fatorial $5 \times 2 \times 2$, com quatro repetições. Os tratamentos consistiram em cinco níveis de reposição hídrica (100, 75, 50, 25 e 0\%, com base na umidade do solo na capacidade de campo) combinados sem e com aplicação de fertilizante nitrogenado ( 0 e $100 \mathrm{~kg} \mathrm{ha}^{-1}$ de $\mathrm{N}$ ) na forma de ureia, em dois ciclos de cultivo. A reposição hídrica promoveu aumento do rendimento bruto de açúcar até 59,34\%, atingindo valores de até 31,60 t ha ${ }^{-1}$. Já a reposição hídrica combinada com a adubação nitrogenada promoveu aumento linear do rendimento bruto de açúcar.

Palavras-chave: Saccharum officinarum L., irrigação, nitrogênio, rendimento.

\footnotetext{
${ }^{1}$ Eng. Agrônomo, Doutorando em Ciências Agrárias - Agronomia, IFGoiano - Campus Rio Verde, Rio Verde GO, Rodovia Sul Goiana, Km 01, CEP: 75.901-170, Rio Verde - GO, e-mail: nelmiciofurtado@gmail.com

${ }^{2}$ Eng. Agrônomo, Doutorando em Ciências Agrárias - Agronomia, Instituto Federal Goiano - Campus Rio Verde, Rio Verde-GO, e-mail: fernandonobrecunha@hotmail.com

3 Eng. Agrônomo, Prof. Dr. em Agronomia, IFGoiano - Campus Rio Verde, Rio Verde - GO, e-mail: marconibt@gmail.com

${ }^{4}$ Eng. Agrícola, Prof. Dr. em Engenharia Agrícola, IFGoiano - Campus Rio Verde, Rio Verde - GO, e-mail: fredalsoares@hotmail.com

${ }^{5}$ Eng. Agrícola, Dr. em Ciências Agrárias - Agronomia, Instituto Federal Goiano - Campus Rio Verde, Rio Verde - GO, e-mail: vmaqrquesvidal@gmail.com

${ }^{6}$ Eng. Ambiental, Dr. em Ciências Agrárias - Agronomia, IFGoiano - Campus Rio Verde, Rio Verde - GO, Rio Verde-GO, e-mail: wilker.alves.morais@gmail.com
} 


\section{WATER REPLACEMENT AND NITROGEN FERTILIZATION ON SUGARCANE VIA SUBSURFACE DRIP: CANE PLANT AND RATOON ABSTRACT}

Improving the quality of sugarcane can be obtained with the use of irrigation, which in addition to increasing the productivity of sugarcane, promotes improvements in the quality indices of the raw material but several factors can affect the quality end so the sugarcane irrigated depends on the amount of water applied, the combined handling of irrigation with the right amount of fertilizer. Starting from the hypothesis that there is interaction between water replacement and nitrogen fertilization in the different cycles of cultivation, this work aimed to follow up on the different stages of growth and development of sugarcane, submitted to different water replacements and nitrogen fertilization via subsurface drip under cane plant and ratoon conditions. The experimental design was a randomized block analyzed in a factorial $5 \times 2 \times 2$, with four replications. The treatments consisted of five levels of water replacement $(100,75$, 50,25 and $0 \%$, based on soil moisture at field capacity) combined with and without application of nitrogen fertilizer ( 0 and $100 \mathrm{~kg} \mathrm{ha}^{-1} \mathrm{~N}$ ) in the form of urea in two cycles of cultivation. The water replacement promoted an increase in the gross sugar yield up to $59.34 \%$, reaching values of up to $31,60 \mathrm{t} \mathrm{ha}^{-1}$. The water replacements combined with the nitrogen fertilization promoted a linear increase in the gross sugar yield.

Keywords: Saccharum officinarum L., irrigation, nitrogen, yield.

\section{INTRODUÇÃO}

A cana-de-açúcar é uma cultura que apresenta certa tolerância ao estresse hídrico, porém, responde a irrigação (SINGH et al., 2007). O incremento na produtividade da canade-açúcar com o uso da irrigação é bastante conhecido (WIEDENFELD; ENCISO, 2008; GAVA et al., 2011). No entanto, o aprimoramento das técnicas de manejo torna-se necessário para atingir a máxima eficiência no uso dos recursos hídricos, visando máxima produtividade com o menor volume de água utilizado. Segundo Gava et al. (2011), o crescimento e o desenvolvimento das plantas são afetados tanto pela falta quanto o excesso do suprimento de água. O cultivo irrigado da cana-de-açúcar proporciona melhorias no ambiente de produção (CARR; KNOX, 2011), porém para o uso eficiente da água pela canade-açúcar é fundamental identificar a necessidade hídrica responsável pelas máximas produções (WIEDENFELD; ENCISO, 2008).

O manejo da irrigação em cana-deaçúcar vem se consolidando como uma ferramenta importante para elevar a produtividade da cultura, resultando em aumentos na produção de colmos e de açúcar
(SOARES et al., 2004; DALRI; CRUZ, 2008). Dentre os métodos de irrigação utilizados para suprir a necessidade hídrica da cana-de-açúcar, destaca-se o sistema de gotejamento. A irrigação por gotejamento permite o controle preciso da água fornecida em pequenas quantidades e alta frequência diretamente para a zona da raiz, possibilitando a manutenção das condições favoráveis de água para a proliferação de raízes no volume de solo parcialmente umedecido (SOUZA et al., 2009).

Para a cana-de-açúcar, os períodos em que o déficit hídrico pode resultar em maiores danos à produtividade da cultura são nos estádios de perfilhamento e de grande crescimento, onde mesmo uma pequena redução na disponibilidade de água do solo pode afetar tanto a divisão celular quanto o alongamento celular em cana-de-açúcar (INMAN-BAMBER; SMITH, 2005). Assim, um conhecimento adequado de como os vegetais respondem a tal estresse abiótico e um dos pré-requisitos para escolher tanto a melhor variedade como as melhores práticas de manejo, visando, sobretudo, aperfeiçoar a exploração dos recursos naturais (SMIT; SINGELS, 2006). Farias et al. (2008) citam que 
a irrigação pode mitigar ou anular os efeitos danosos da deficiência hídrica

$\mathrm{Na}$ literatura existem trabalhos que mostram a importância do $\mathrm{N}$ na cultura da canade-açúcar (OLIVEIRA et al., 2013; FRANCO et al., 2011). Wiedenfeld e Enciso (2008), em estudos desenvolvidos com a aplicação de $\mathrm{N}$ por irrigação de gotejo subsuperficial em canade-açúcar verificaram que os resultados positivos da fertilização nitrogenada foram proporcionais a quantidade de água aplicada.

Pesquisas em várias regiões produtoras do mundo e do Brasil, com diferentes variedades de cana-de-açúcar, têm mostrado o efeito da irrigação no aumento da produtividade, além disso, a adubação nitrogenada destaca-se como uma das práticas culturais de maior demanda de pesquisas para a cana-de-açúcar, uma vez que os estudos sobre $\mathrm{N}$ apresentam resultados muito variáveis e muitas vezes até contraditórios. Neste contexto, formulou-se a hipótese de que existe interação entre reposição hídrica e adubação nitrogenada nos diferentes ciclos de cultivo. Dessa forma, objetivou-se com este trabalho acompanhar nas diferentes fases de crescimento e desenvolvimento as variáveis biométricas altura de planta e diâmetro de colmos além do rendimento bruto de açúcar da cana-de-açúcar, submetida a diferentes reposições hídricas e adubação nitrogenada via gotejamento subsuperficial nas condições de cana-planta e soca.

\section{MATERIAL E MÉTODOS}

O experimento foi conduzido em condições de campo, no período de março de 2011 a maio de 2012 (Cana-planta) e de junho de 2012 a junho de 2013 (Cana-soca), na estação experimental do Instituto Federal Goiano - Campus Rio Verde - GO. As coordenadas geográficas do local de instalação são $17^{\circ} 48^{\prime} 28^{\prime \prime}$ S e $50^{\circ} 53^{\prime} 57^{\prime \prime} \mathrm{O}$, com altitude média de $720 \mathrm{~m}$ ao nível do mar. O clima da região é classificado conforme Köppen e Geiger (1928), como Aw (tropical), com chuva nos meses de outubro a maio, e com seca nos meses de junho a setembro. A temperatura média anual varia de 20 a $35{ }^{\circ} \mathrm{C}$ e as precipitações variam de 1.500 a $1.800 \mathrm{~mm}$ anuais e o relevo é suave ondulado ( $6 \%$ de declividade).

Tabela 1. Características físico-hídricas e químicas do solo da área experimental, nas camadas de 00,20 e $0,20-0,40 \mathrm{~m}$ de profundidade.

\begin{tabular}{|c|c|c|c|c|c|c|c|c|c|c|}
\hline \multicolumn{11}{|c|}{ Características físico-hídricas } \\
\hline \multirow{2}{*}{$\begin{array}{c}\text { Camada } \\
(\mathrm{m})\end{array}$} & \multicolumn{3}{|c|}{ Granulometria $\left(\mathrm{g} \mathrm{kg}^{-1}\right)$} & $\theta_{\mathrm{CC}}$ & $\theta_{\mathrm{PMP}}$ & \multirow{2}{*}{$\begin{array}{c}\text { Ds } \\
\mathrm{g} \mathrm{cm}^{-3}\end{array}$} & \multirow{2}{*}{$\begin{array}{c}\mathrm{PT} \\
\mathrm{cm}^{3} \mathrm{~cm}^{-3} \\
\end{array}$} & \multirow{2}{*}{\multicolumn{3}{|c|}{ Classificação textural }} \\
\hline & Areia & Silte & Argila & \multicolumn{2}{|r|}{$\%$} & & & & & \\
\hline $0-0,20$ & 458,3 & 150,2 & 391,5 & 51,83 & 30,5 & 1,27 & 0,55 & \multicolumn{3}{|c|}{ Franco Argiloso } \\
\hline $0,20-0,40$ & 374,9 & 158,3 & 466,8 & 55 & 31,33 & 1,28 & 0,51 & \multicolumn{3}{|c|}{ Argila } \\
\hline \multicolumn{11}{|c|}{ Características químicas } \\
\hline Camada & $\mathrm{pH}$ & $\mathrm{MO}$ & $\mathrm{P}$ & K & $\mathrm{Ca}$ & $\mathrm{Mg}$ & $\mathrm{H}+\mathrm{Al}$ & $\mathrm{S}$ & CTC & $\mathrm{V}$ \\
\hline$(\mathrm{m})$ & $\begin{array}{c}\mathrm{em} \\
\mathrm{H}_{2} \mathrm{O}\end{array}$ & $\mathrm{g} \mathrm{kg}^{-1}$ & $\mathrm{mg} \mathrm{dm} \mathrm{m}^{-3}$ & & & $n$ & nol dm & & & $(\%)$ \\
\hline $0,00-0,20$ & 6,2 & 63,42 & 7,06 & 2,04 & 20,40 & 16,80 & 57,75 & 41,80 & 99,55 & 41,99 \\
\hline $0,20-0,40$ & 6,6 & 44,47 & 2,65 & 4,09 & 14,40 & 13,20 & 44,55 & 31,69 & 76,24 & 41,57 \\
\hline
\end{tabular}

$\theta_{\mathrm{CC}}$, capacidade de campo $(10 \mathrm{KPa})$; $\theta_{\mathrm{PMP}}$, ponto de murcha permanente $(1.500 \mathrm{KPa})$; Ds, densidade do solo; PT, porosidade total; pH em água destilada. P e K, extrator Mehlich ${ }^{-1}$. M.O - Matéria orgânica. V - Saturação por bases.

O delineamento experimental utilizado foi o de blocos ao acaso, analisado em esquema fatorial $5 \times 2 \times 2$, com quatro repetições. Os tratamentos consistiram em cinco níveis de reposição hídrica $(100,75,50,25$ e $0 \%$, com base na umidade do solo na capacidade de campo) combinados sem e com aplicação de fertilizante nitrogenado (0 e $100 \mathrm{~kg} \mathrm{ha}^{-1}$ de $\mathrm{N}$ ) na forma de ureia, em dois ciclos de cultivo.

Para a condução do ensaio, selecionouse a área de $2300 \mathrm{~m}^{2}$, que antes do plantio da cana-de-açúcar, foi cultivada por 20 anos com pastagem. O preparo inicial do solo consistiu de gradagem prévia com o intuito de eliminar a 
vegetação existente, distribuição de calcário dolomítico, na dosagem de 2,0 t ha ${ }^{-1}$, com base nos resultados da análise de solo, com intuito de elevar a saturação por bases, conforme recomendado por Sousa e Lobato (2004). O corretivo foi distribuído por meio de distribuidora de calcário tratorizada, e posteriormente se realizou outra gradagem com o propósito de incorporar o calcário e destorroar o solo. Por último, realizou-se a gradagem de nivelamento.

O plantio da cana-de-açúcar foi realizado em março de 2011, utilizando-se a variedade RB 85-5453, que apresenta como características principais alto teor de açúcar e precocidade. As parcelas experimentais foram compostas por três sulcos de linha dupla (plantio “em W") com espaçamento de 1,4 x 0,4 $\mathrm{m}$ entre linhas e $8 \mathrm{~m}$ de comprimento, totalizando 43,2 $\mathrm{m}^{2}$ de área total por parcela.

Nos tratamentos com reposição hídrica, foi utilizado o método de irrigação por gotejamento subsuperficial. $\mathrm{O}$ tubo gotejador foi enterrado a $0,20 \mathrm{~m}$ de profundidade da superfície do solo, no meio da linha dupla, tendo o mesmo as seguintes características: modelo Dripnet PC 16150 com parede delgada, pressão de serviço de 1,0 bar, vazão nominal de $1,0 \mathrm{~L} \mathrm{~h}^{-1}$ e espaçamento entre gotejadores de $0,50 \mathrm{~m}$.

A irrigação foi conduzida com base em tensiometria digital de punção com sensibilidade de $0,1 \mathrm{kPa}$, sendo as hastes tensiométricas instaladas nas profundidades de 0,20 e 0,40 e distâncias de $0,15,0,30,0,45$ e $0,60 \mathrm{~m}$ do tubo gotejador, com leitura do potencial matricial do solo $(\Psi \mathrm{m})$ registrada diariamente. Para determinar a necessidade de irrigação, utilizou-se tensão crítica de $40 \mathrm{kPa}$.

As características físico-hídricas do solo foram determinadas obtendo-se, assim, a curva de retenção de água no solo, a partir do uso do software RETEC (van Genuchten, 2009), que possibilita gerar os parâmetros empíricos de ajuste da equação de van Genuchten (1980), convertendo o $\Psi \mathrm{m}$ mensurado em campo em conteúdo de água no solo $(\theta)$, conforme a equação de van Genutchen (1980) a seguir:

$$
\theta=\theta_{r}+\frac{\theta_{s}-\theta_{r}}{\left[1+\left(\alpha \times\left|\psi_{m}\right|\right)^{n}\right]^{m}}
$$

em que:

$\theta$ - conteúdo de água no solo, $\mathrm{cm}^{3} \mathrm{~cm}^{-3}$;

$\Psi_{\mathrm{m}}$ - potencial mátrico, $\mathrm{kPa}$;

$\theta_{\mathrm{s}}$ - umidade do solo saturado, $\mathrm{cm}^{3} \mathrm{~cm}^{-3}$;

$\theta_{r}$ - umidade do solo residual, $\mathrm{cm}^{3} \mathrm{~cm}^{-3}$; e

$\alpha, \mathrm{n}, \mathrm{m}$ - parâmetros de ajuste empíricos.

Os resultados diários do conteúdo de água no solo foram utilizados para determinar o volume de água aplicado para cada reposição hídrica, sendo que nos tratamentos de 100\% baseou-se na elevação da umidade do solo até a capacidade de campo. Para os demais tratamentos, foram aplicadas lâminas de acordo com a porcentagem prevista de reposição hídrica.

A área experimental foi quimicamente corrigida nos sulcos de plantio conforme o resultado da análise de solo e recomendação de Sousa e Lobato (2004), com aplicação de 120 $\mathrm{kg} \mathrm{ha}^{-1}$ de $\mathrm{P}_{2} \mathrm{O}_{5}$ (superfosfato simples) e $80 \mathrm{~kg}$ $\mathrm{ha}^{-1}$ de $\mathrm{K}_{2} \mathrm{O}$ (cloreto de potássio). Na condição de cana-planta e cana-soca, nas parcelas em que foi prevista a aplicação de $100 \mathrm{~kg} \mathrm{ha}^{-1}$ de $\mathrm{N}$ (ureia), este foi aplicado totalmente via água de irrigação (Fertirrigação), parcelado em dez aplicações ao longo do ciclo da cultura.

A partir dos dados climatológicos do período experimental, foi elaborada a estimativa do balanço hídrico decendial para a cana-de-açúcar em manejo de sequeiro (Figura 1), empregando-se o método de Thornthwaite e Mather (1955), sendo que a evapotranspiração de referência $\left(\mathrm{ET}_{0}\right)$ foi calculada segundo a equação de Penman-Monteith-FAO/56 (ALLEN et al., 1998). 


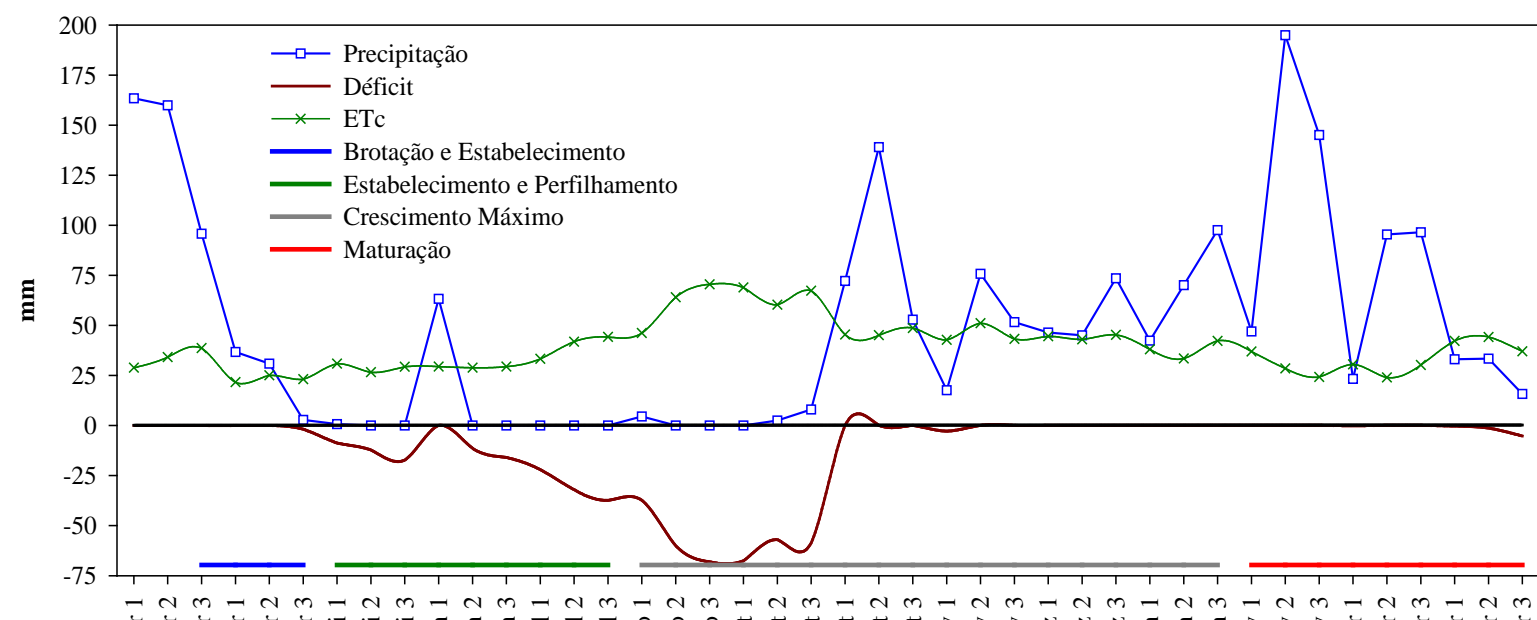

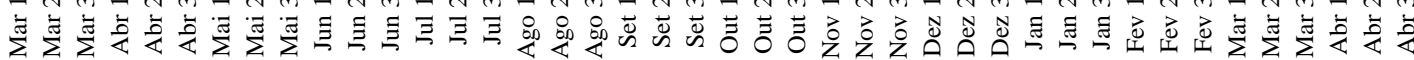

2011

2012

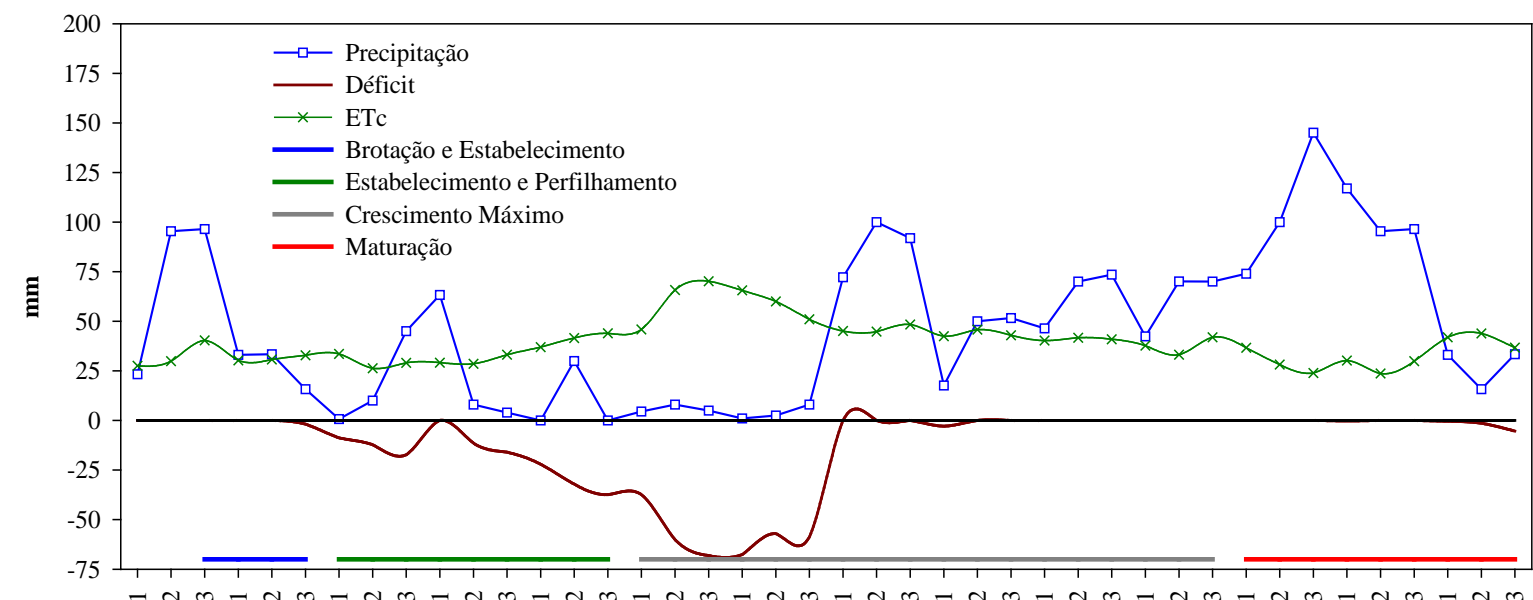

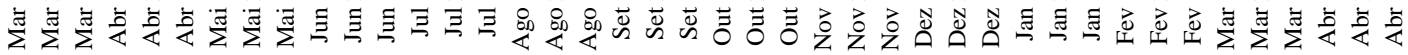

2012

2013

Figura 1. Balanço hídrico da cana-de-açúcar em manejo de sequeiro. DEF - Déficit hídrico. ETc Evapotranspiração da cultura. Fases da cultura (adaptado de DOORENBOS; KASSAM, 1994): Brotação e Estabelecimento $(\mathrm{Kc}=0.6)$; Estabelecimento e Perfilhamento $(\mathrm{Kc}=0.9$ a 1.1$)$; Crescimento Máximo $(\mathrm{Kc}=$ 1.3); e Maturação (Kc = 0.7 a 0.9). Fonte: Estação Normal INMET - Rio Verde - GO.

As características morfológicas foram avaliadas mensalmente nas linhas centrais de cada parcela, quantificando: Altura de Planta (AP); Diâmetro de colmo (DC). A altura da planta foi mensurada com auxílio de fita métrica, a partir do solo até o colarinho da folha +1 (folha +1 é aquela que se pode visualizar completamente o colarinho), e expresso em cm; O diâmetro de colmos foi determinado pelo auxílio do paquímetro no terço médio da planta, e expresso em mm.

Depois das avaliações, os dados foram agrupados segundo os estádios de desenvolvimento da cana-de-açúcar, ou seja, o valor de cada dado representa uma média de medidas feitas mensalmente, dentro de cada fase, conforme Diola e Santos (2012), em que os estádios de desenvolvimento da cana-deaçúcar se dividem em quatro fases, a saber: (a) Brotação e estabelecimento da cultura; (b) Perfilhamento: estende-se desde o final da brotação até 120 dias após o plantio; (c) Desenvolvimento vegetativo e crescimento dos colmos: inicia-se logo após a fase de perfilhamento até 270 dias após o plantio; (d) Maturação: fase de síntese e acúmulo de açúcar, que dura de 270-300 até 360 dias após o plantio.Sendo que neste estudo as avaliações 
iniciaram após o estádio (a) Brotação e estabelecimento da cultura, além disso, cada estádio foi chamado de fase: (b) Fase I perfilhamento; (c) Fase II - crescimento máximo; (d) Fase III - maturação.

Foi realizado o monitoramento do ${ }^{\circ}$ Brix da cana-de-açúcar em campo, nas três últimas semanas antes da colheita em cada ciclo de cultivo. Para a determinação racional do ponto de colheita da cana-de-açúcar, utilizou-se o parâmetro conhecido como Índice de Maturação (IM) determinado em campo, utilizando-se um refratômetro portátil. Os valores de IM são: (a) menor que 0,60 para cana verde; (b) entre 0,60 e 0,85 para cana em processo de maturação; (c) entre 0,85 e 1 para cana madura; e (d) maior que 1 para cana em processo de declínio de sacarose (ROSSETO, 2012). No momento em que se atingiu Índice de Maturação (IM = ${ }^{\circ}$ Brix do Ápice $/{ }^{\circ}$ Brix da Base) entre 0,9 e 0,95, foi realizada a colheita.

A produtividade de colmos foi determinada através da pesagem total dos colmos presentes na área útil das respectivas parcelas sendo quantificado o peso dos colmos presentes nas duas linhas centrais de cada parcela, cujo valor foi extrapolado para $\mathrm{t} \mathrm{ha}^{-1}$. Para tanto, realizou-se o corte o mais rente possível do solo. Os colmos foram então despalhados e tiveram o ponteiro destacado. Em seguida, foram pesados em balança digital tipo gancho, marca Soil Control (precisão = 0,02 kg), com capacidade de $50 \mathrm{~kg}$.

Os rendimentos brutos de açúcar e de álcool foram calculados baseados nestes resultados, de acordo com metodologia descrita por Caldas (1998):

$$
\mathrm{RBAç}=\left(\frac{\mathrm{PCC} * \mathrm{PC}}{100}\right)
$$

em que:

RBAç - rendimento bruto de açúcar em t ha-1; PCC - quantidade de açúcar bruto em \% contido nos colmos e determinada em laboratório; e PC - produção de colmos em $t h^{-1}$.
Os resultados foram submetidos à análise da variância pelo teste $\mathrm{F}$ ao nível de $5 \%$ de probabilidade, e em casos de significância, foi realizada a análise de regressão polinomial linear e quadrática para os níveis de reposição hídrica, enquanto para o fator aplicação de nitrogênio e ciclo as médias foram comparadas entre si pelo teste Tukey à 5\% de probabilidade, utilizando-se o programa estatístico SISVAR ${ }^{\circledR}$ (FERREIRA, 2011).

\section{RESULTADOS E DISCUSSÃO}

As fases da cana-de-açúcar analisadas com relação a variável AP, apresentaram efeito significativo isoladamente para todas as fases com relação aos fatores $\mathrm{RH}$ e C, e para o fator $\mathrm{N}$ na fase III. Observa-se que as interações entre estes fatores apresentaram significância para $\mathrm{RH} \times \mathrm{C}$ na fase II e $\mathrm{N} \times \mathrm{C}$ na fase III. Estes resultados mostram que a $\mathrm{RH}$ e o $\mathrm{C}$ tiveram efeito durante todas as fases de crescimento e desenvolvimento da cana-de-açúcar, sendo que está resposta apresentou dependência entre o fator RH e C na fase II; $\mathrm{N}$ e C na fase III. O CV não apresentou variações elevadas para a variável AP, variou de 11,47 a 2,93\%, pode-se observar ainda, que no decorrer do crescimento e desenvolvimento, o CV tendeu a diminuir da fase I para a fase III, indicando menor variação na AP verificados em função dos tratamentos, evidenciando dessa forma, uma boa precisão experimental na determinação da AP.

A AP analisada isoladamente quanto ao fator $C$ na fase I apresentou a média superior em 6,58\% para cana-planta quando comparado à cana-soca, conforme Tabela 2.

Estes resultados evidenciam que a condição de cana-planta propicia maior crescimento das plantas na fase inicial de cultivo por consumir as reservas oriundas do tolete. Farias et al. (2008) obtiveram, para a cana-de-açúcar irrigada, altura máxima de $152,80 \mathrm{~cm}$ aos 193,85 dias e taxa de crescimento em altura, aos 280 dias após o plantio (DAP), de $0,5457 \mathrm{~cm} \mathrm{dia}^{-1}$.

Tabela 2. Teste de médias para o fator C na fase I da cana-de-açúcar, Rio Verde, Goiás, safras 2011/2012 e 2012/2013. 


\begin{tabular}{cc}
\hline \multirow{2}{*}{$\mathrm{C}$} & Altura de Planta (AP) \\
\cline { 2 - 2 } & Fase I \\
\hline Cana-planta & Médias (cm) \\
Cana-soca & $296,1322 \mathrm{a}$ \\
\hline
\end{tabular}

* Médias seguidas de mesma letra nas colunas não diferem entre si segundo teste Tukey a 5\% de probabilidade; FV - Fontes de variação; C - ciclo.

A AP quando analisada isoladamente na fase I e III em função da RH, adequaram-se a relações lineares, com $\mathrm{R}^{2}$ de 91,09 e 97,05\%, indicando que apenas 8,91 e 2,95\% das variações não são explicadas pela variação da RH aplicada. Mediante os resultados estimados para a Fase I e III, pode-se observar que a cada $25 \%$ de $\mathrm{RH}$, houve incremento de 1,33 e 7,47 cm, que correspondem respectivamente a 3,74 e $2,47 \%$, totalizando incrementos de até 5,33 e $29,88 \mathrm{~cm}$, que correspondem respectivamente 14,96 e $9,88 \%$ para RH de $100 \%$, conforme Figura 2.

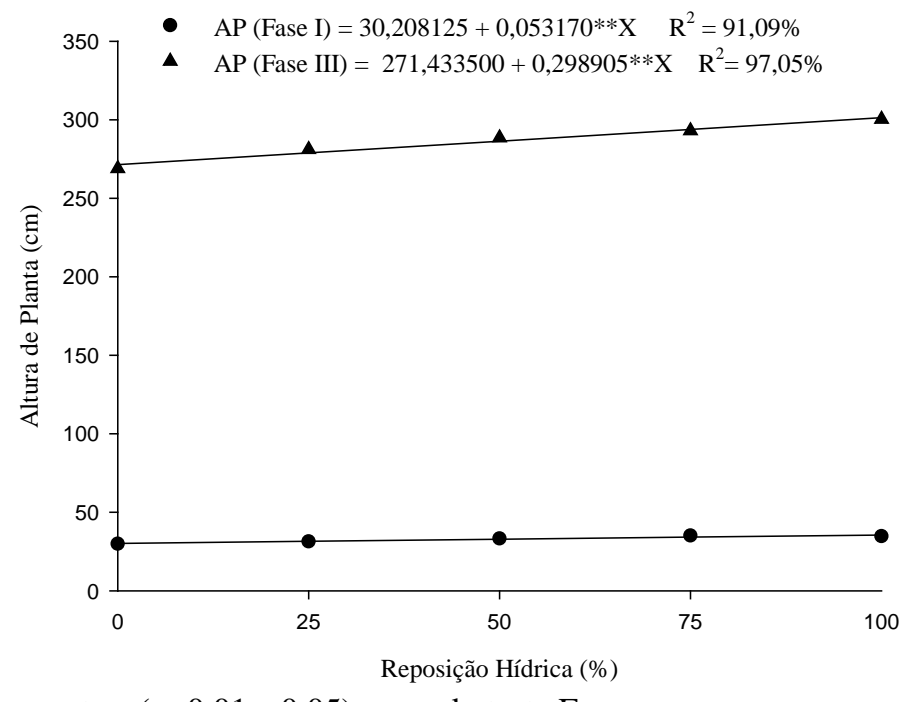

** $\mathrm{e}^{*}$ significativo respectivamente a $(\mathrm{p}<0,01$ e 0,05$)$ segundo teste $\mathrm{F}$.

Figura 2. Altura de planta em função da Reposição Hídrica na Fase I e III da cana-de-açúcar, Rio Verde, Goiás, safras 2011/2012 e 2012/2013.

Na fase III, o desdobramento do fator $\mathrm{N}$ dentro $\mathrm{C}$ apresentou efeito significativo para Cana-soca, sendo a maior média foi observada em ComN. Já para o desdobramento de C dentro de $\mathrm{N}$ apresentou efeito significativo para ComN e SemN sendo que as maiores médias foram observadas em Cana-planta, conforme Tabela 3.

Tabela 3. Teste de media para o desdobramento do fator $\mathrm{N}$ x C na Fase III de cultivo da cana-deaçúcar, Rio Verde, Goiás, safras 2011/2012 e 2012/2013.

\begin{tabular}{|c|c|c|}
\hline \multicolumn{3}{|c|}{ Altura de Plantas (AP) } \\
\hline \multirow{2}{*}{ Fase III } & Cana-planta & Cana-soca \\
\hline & \multicolumn{2}{|c|}{ Médias (cm) } \\
\hline ComN & 294,3465 A & $283,4422 \mathrm{Ba}$ \\
\hline SemN & 297,9180 A & $269,8085 \mathrm{Bb}$ \\
\hline
\end{tabular}

* Médias seguidas de mesma letra minúscula nas colunas, e maiúscula nas linhas, não diferem entre si segundo teste Tukey a 5\% de probabilidade, FV - Fontes de variação.

O desdobramento da AP para fator $\mathrm{RH}$ dentro de $C$ na fase II adequou-se a relação linear, com $\mathrm{R}^{2}$ de 71,56 e 78,49\%, respectivamente para cana-planta e cana-soca, indicando que 28,44 e $21,51 \%$ das variações não são explicadas pela variação da RH 
aplicada na Fase II. Mediante os resultados estimados para cana-planta e soca, pode-se observar que a cada $25 \%$ de $\mathrm{RH}$, houve o incremento de 1,42 e $1,22 \mathrm{~cm}$, que correspondem respectivamente a 3,11 e 4,88\%, totalizando incrementos de até 5,68 e $4,88 \mathrm{~cm}$, que correspondem respectivamente a 12,44 e 19,52\% para RH de 100\%, conforme Figura 3.

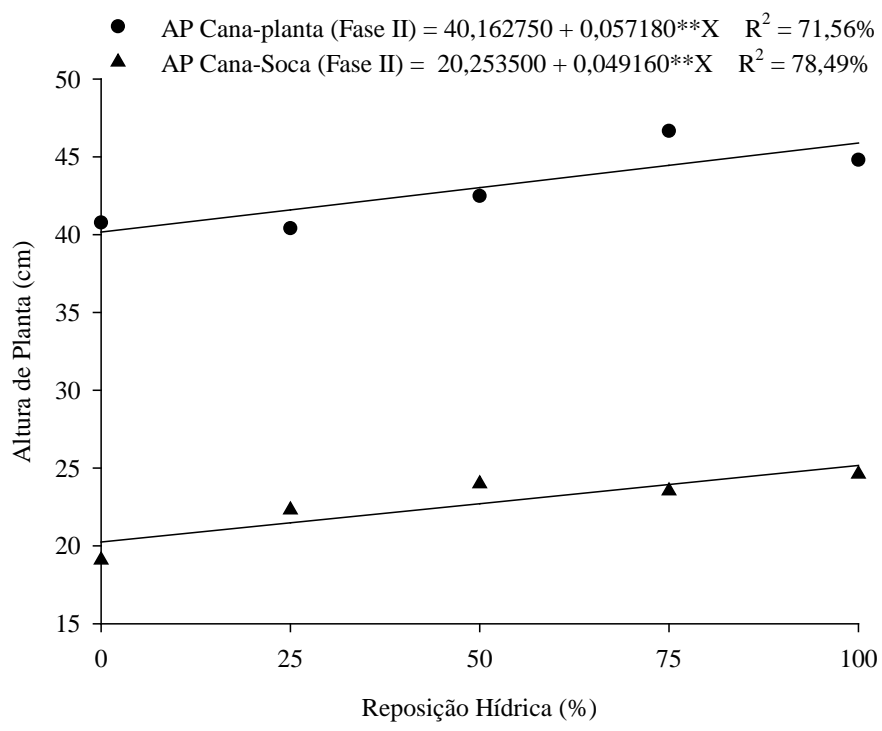

${ }^{* *} \mathrm{e}^{*}$ significativo respectivamente a $(\mathrm{p}<0,01$ e 0,05$)$ segundo teste $\mathrm{F}$.

Figura 3. Altura de planta em função da Reposição Hídrica na Fase II da cana-de-açúcar, Rio Verde, Goiás, safras 2011/2012 e 2012/2013.

Na fase II, o desdobramento do fator C dentro $\mathrm{RH}$ apresentou efeito significativo a partir de $25 \%$ de $\mathrm{RH}$, sendo que as maiores médias foram observadas em Cana-soca, com o aumento de 9,98; 14,49; 9,46 e 12,85\%, respectivamente para 25, 50, 75 e $100 \%$ de $\mathrm{RH}$ quando comparado a Cana-planta, conforme Tabela 4. Segundo Silva et al. (2008), a variação na altura da planta é indicativo de tolerância ou suscetibilidade da cana-de-açúcar ao déficit hídrico. Para Cintra et al. (2008), afirmam que os estágios intermediários (desenvolvimento) respondem à lâmina de irrigação, mas o déficit hídrico não causa tantos prejuízos à produtividade quanto os primeiros: portanto, o intervalo de irrigação pode ser ampliado, porém a lâmina de água deve ser aumentada. Com o suprimento adequado de água, esse estágio é atingido rapidamente e a altura final da cana também é maior.

Tabela 4. Teste de media para o desdobramento do fator $\mathrm{C}$ x RH na Fase II de cultivo da cana-deaçúcar, Rio Verde, Goiás, safras 2011/2012 e 2012/2013.

\begin{tabular}{lrrrr}
\multirow{2}{*}{$\mathrm{C}$} & \multicolumn{5}{c}{ Altura de planta (AP) } \\
\cline { 2 - 5 } & $25 \%$ & $50 \%$ & \multicolumn{1}{c}{$75 \%$} \\
\hline \\
\cline { 2 - 5 } Cana-planta & $122,2250 \mathrm{~b}$ & $121,5350 \mathrm{~b}$ & $132,7325 \mathrm{~b}$ & $135,4425 \mathrm{~b}$ \\
Cana-soca & $135,7900 \mathrm{a}$ & $142,1375 \mathrm{a}$ & $146,6112 \mathrm{a}$ & $155,4250 \mathrm{a}$ \\
\hline
\end{tabular}

* Médias seguidas de mesma letra nas colunas não diferem entre si segundo teste Tukey a 5\% de probabilidade, FV Fontes de variação; C - ciclo.

As fases da cana-de-açúcar analisadas com relação a variável DC, apresentaram efeito significativo isoladamente para todas as fases com relação aos fatores RH e C, e para o fator $\mathrm{N}$ na fase III. Quando analisada a interação entre estes fatores houve significância para $\mathrm{RH}$ x C na fase I. Estes resultados mostram que a $\mathrm{RH}$ e o $\mathrm{C}$ tiveram efeito durante todas as fases de crescimento e desenvolvimento da cana-de-açúcar, sendo que está resposta 
apresentou dependência na fase I, conforme Tabela 5.

O CV não apresentou variações elevadas para a variável DC, variou de 8,60 a 4,99\%, pode-se observar ainda, que no decorrer do crescimento e desenvolvimento, o CV tendeu a diminuir da fase I para a fase III, indicando menor variação na DC verificados em função dos tratamentos, evidenciando dessa forma, uma boa precisão experimental na determinação do DC. Dantas Neto et al. (2006) também observaram efeito significativo do diâmetro do colmo em função da disponibilidade hídrica.

$\mathrm{O}$ DC analisado isoladamente quanto ao fator $\mathrm{C}$ na fase II $\mathrm{e}$
III, apresentou a média superior em 8,74 e $10,22 \%$, respectivamente para fase II e III, para cana-planta quando comparado a cana-soca, e quanto ao fator $\mathrm{N}$ na fase III, apresentou a média superior em $3,34 \%$ para $\mathrm{ComN}$ quando comparado a SemN, conforme Tabela 7.

Apesar destes resultados pode-se dizer que esta redução no DC não foi tão proeminente uma vez que a cana-de-açúcar de um ciclo a outro pode apresentar valores de redução de DC elevados. Já com relação à adubação nitrogenada e notório o maior aproveitamento pelas plantas quando aplicada via água de irrigação.

Tabela 5. Teste de médias para o fator C na fase II e III, e fator N na fase III da cana-de-açúcar, Rio Verde, Goiás, safras 2011/2012 e 2012/2013.

\begin{tabular}{|c|c|c|}
\hline \multirow{2}{*}{$\mathrm{C}$} & \multicolumn{2}{|c|}{ Diâmetro de Colmos (DC) } \\
\hline & Fase II & Fase III \\
\hline & \multicolumn{2}{|c|}{ Médias (mm) } \\
\hline Cana-planta & $30,8567 \mathrm{a}$ & $35,4200 \mathrm{a}$ \\
\hline Cana-soca & $28,1587 \mathrm{~b}$ & $31,7995 \mathrm{~b}$ \\
\hline $\mathrm{N}$ & \multicolumn{2}{|c|}{ Fase III } \\
\hline ComN & \multicolumn{2}{|c|}{34,1817 a } \\
\hline SemN & \multicolumn{2}{|c|}{$33,0377 \mathrm{~b}$} \\
\hline
\end{tabular}

* Médias seguidas de mesma letra nas colunas não diferem entre si segundo teste Tukey a 5\% de probabilidade. FV Fontes de variação; C - ciclo.

O DC quando analisado isoladamente para o fator RH na fase II e III, adequaram-se a relações lineares, com $\mathrm{R}^{2}$ de 84,37 e $90,59 \%$, respectivamente para fase II e III, indicando que 15,65 e 9,41\% das variações não são explicadas pela variação da RH aplicada na fase II e III. Mediante os resultados estimados para fase II e

III, pode-se observar que a cada $25 \%$ de $\mathrm{RH}$, houve o incremento de 1,86 e $0,80 \mathrm{~mm}$, que correspondem respectivamente a 1,86 e 2,28\%, totalizando incrementos de até 7,44 e $3,20 \mathrm{~mm}$, que correspondem respectivamente a 7,44 e 9,12\% para $\mathrm{RH}$ de $100 \%$, conforme Figura 4. 


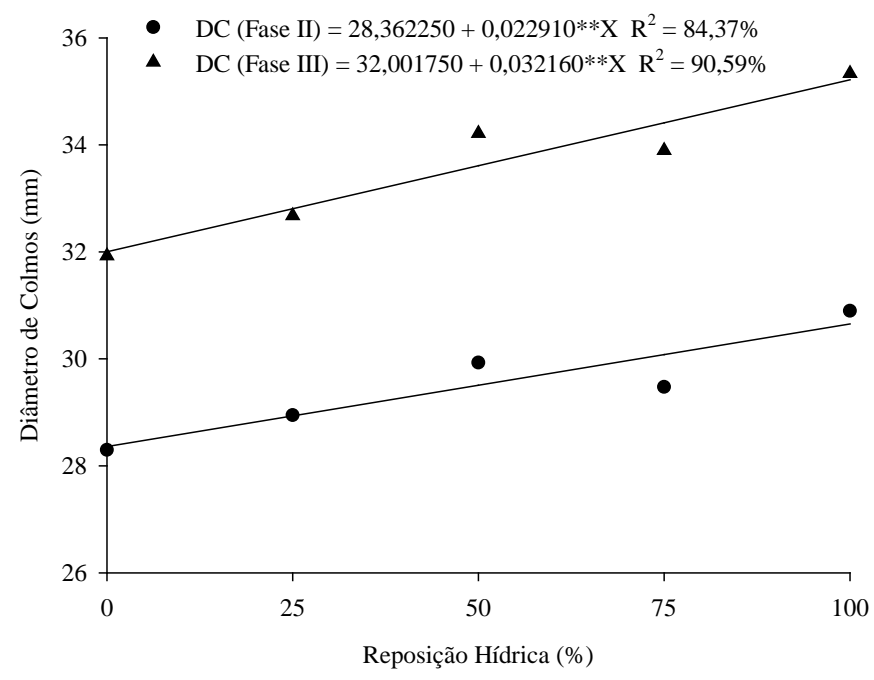

${ }^{* *} \mathrm{e}^{*}$ significativo respectivamente a $(\mathrm{p}<0,01$ e 0,05$)$ segundo teste $\mathrm{F}$.

Figura 4. Diâmetro de colmos em função da Reposição Hídrica na Fase II e III da cana-de-açúcar, Rio Verde, Goiás, safras 2011/2012 e 2012/2013.

O desdobramento do DC para fator $\mathrm{RH} x$ $\mathrm{C}$ na fase I, adequou-se a relação linear, com $\mathrm{R}^{2}$ de $93,71 \%$, indicando que apenas $6,29 \%$ das variações não são explicadas pela variação da RH aplicada. Mediante os resultados estimados, pode-se observar que a cada $25 \%$ de $\mathrm{RH}$, houve o incremento de $1,21 \mathrm{~mm}$, que corresponde a $6,06 \%$, totalizando incremento de até $4,84 \mathrm{~mm}$, que corresponde a $24,24 \%$ para $\mathrm{RH}$ de $100 \%$, conforme Figura 5.

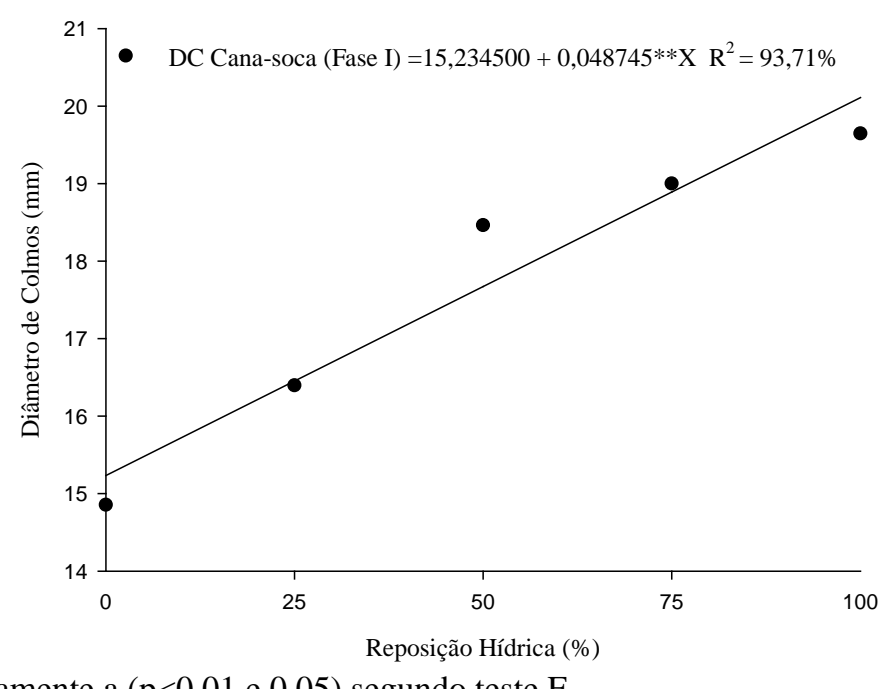

** $\mathrm{e}^{*}$ significativo respectivamente a $(\mathrm{p}<0,01$ e 0,05$)$ segundo teste $\mathrm{F}$.

Figura 5. Diâmetro de colmos em função da Reposição Hídrica na fase I da cana-de-açúcar, Rio Verde, Goiás, safras 2011/2012 e 2012/2013.

Na fase I o desdobramento do fator C dentro $\mathrm{RH}$, apresentou efeito significativo para todos os níveis de $\mathrm{RH}$, sendo que as maiores médias foram observadas em Cana-planta, com o aumento de 34,26; 30,63; 19,34; 16,86 e $17,37 \%$, respectivamente para $0,25,50,75$ e
100\% de RH quando comparado a Cana-soca, conforme Tabela 6. Os resultados obtidos corroboram com Moura et al. (2005), que consideram adequados valores de DC acima de $22 \mathrm{~mm}$ para a cana-de-açúcar irrigada.

Tabela 6. Teste de media para o desdobramento do fator C x RH na fase I de cultivo da cana-de-açúcar, Rio Verde, Goiás, safras 2011/2012 e 2012/2013. 


\begin{tabular}{|c|c|c|c|c|c|}
\hline & \multicolumn{5}{|c|}{ Diâmetro de Colmos (DC) } \\
\hline $\mathrm{C}$ & $0 \%$ & $25 \%$ & $50 \%$ & $75 \%$ & $100 \%$ \\
\hline & & & Médias (n & & \\
\hline Cana-planta & $22,6000 \mathrm{a}$ & 23,6362 a & $22,8900 \mathrm{a}$ & $23,6300 \mathrm{a}$ & 23,7775 a \\
\hline Cana-soca & $14,8550 \mathrm{~b}$ & $16,3962 \mathrm{~b}$ & $18,4612 b$ & $19,0000 \mathrm{~b}$ & $19,6462 b$ \\
\hline
\end{tabular}

* Médias seguidas de mesma letra nas colunas não diferem entre si segundo teste Tukey a 5\% de probabilidade. FV Fontes de variação; C - ciclo.

A variável produtiva RBAç apresentou efeito significativo para a interação entre $\mathrm{RH} x$ $\mathrm{C}$ e $\mathrm{RH} \times \mathrm{N}$. Estes resultados evidenciaram relação direta entre a irrigação por gotejamento subsuperficial, o $\mathrm{N}$ aplicado via irrigação na RBAç da cana-de-açúcar. O efeito significativo do nitrogênio nas variáveis produtivas pode ser atribuído ao fornecimento do fertilizante em pequenas doses ao longo do ciclo de cultivo, aumentando a absorção e favorecendo o aproveitamento do nitrogênio, por apresentar sincronismo maior de disponibilização e absorção de nutrientes para as plantas (GAVA et al., 2011). Carvalho et al. (2009) também verificaram aumento no rendimento bruto de açúcar com o aumento do nível de irrigação.

O desdobramento do RBAç para o fator $\mathrm{RH} \times \mathrm{N}$ se adequou respectivamente a relações lineares, com $\mathrm{R}^{2}$ de 86,93 e $71,85 \%$ para Com $\mathrm{N}$ e Sem $\mathrm{N}$, indicando que apenas 13,07 e 28,15\% das variações não são explicadas pela variação da RH. Mediante os resultados estimados, pode-se observar que Com $\mathrm{N}$ a cada $25 \%$ de $\mathrm{RH}$, houve o incremento de $2,41 \mathrm{t} \mathrm{ha}^{-1}$, que correspondem a 25,98\%, conforme a Figura 6. Já para Sem $\mathrm{N}$ o aumento máximo ocorreu na RH de 59,34\% com aumentos de até 31,60 t ha' 1. Dantas Neto et al. (2006) observaram que a adubação de cobertura nas doses de $157 \mathrm{~kg} \mathrm{ha}^{-1}$ de $\mathrm{N}$ mais $148 \mathrm{~kg} \mathrm{ha}^{-1}$ de $\mathrm{K}_{2} \mathrm{O}$, proporcionaram aumentos significativos na qualidade tecnológica da cana-de-açúcar, com acréscimo de $39,8 \%$ para RBAç alcançando um rendimento de $12,58 \mathrm{Mg} \mathrm{ha}^{-1}$ de açúcar. Resultados favoráveis também foram encontrados por Moura et al. (2005).

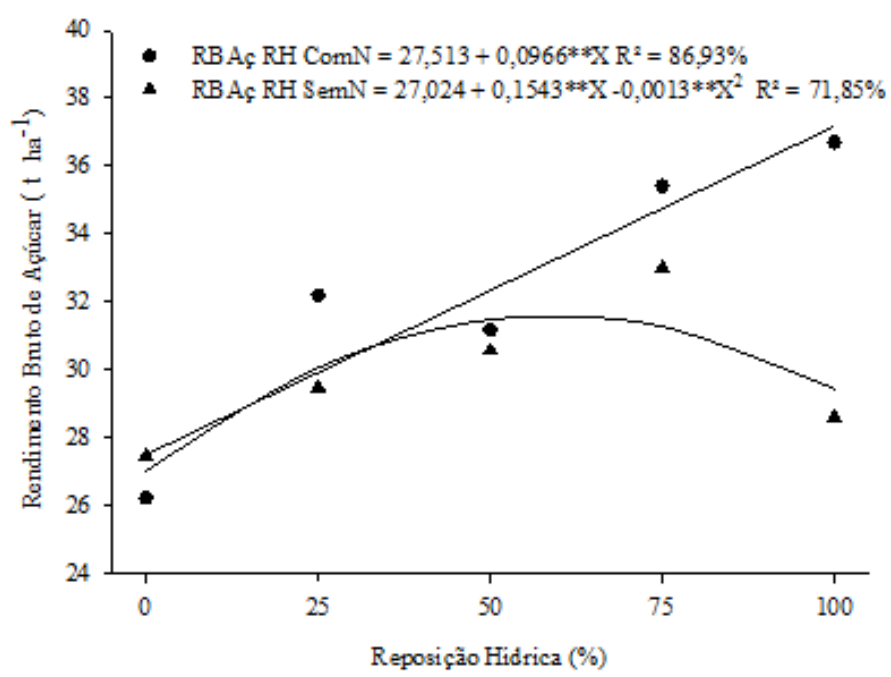

** $\mathrm{e}^{*}$ significativo respectivamente a $(\mathrm{p}<0,01$ e 0,05$)$ segundo teste $\mathrm{F}$.

Figura 6. Rendimento bruto de açúcar em função da Reposição Hídrica x Nitrogênio na cana-de-açúcar, Rio Verde, Goiás, safras 2011/2012 e 2012/2013.

O desdobramento do RBAç para o fator $\mathrm{RH} \times \mathrm{C}$ se adequou respectivamente a relações lineares, com $\mathrm{R}^{2}$ de 76,36 e 93,73\% para Canaplanta e Cana-soca, indicando que apenas 23,64 e 6,27\% das variações não são explicadas pela variação da $\mathrm{RH}$.
Mediante os resultados estimados, pode-se observar que o ponto de máximo para Canaplanta e soca ocorreram respectivamente com $57,18 \quad 100 \%$ de $\mathrm{RH}$, atingindo valores de até 33,33 e 34,42 tha ${ }^{-1}$ (Figura 7). 


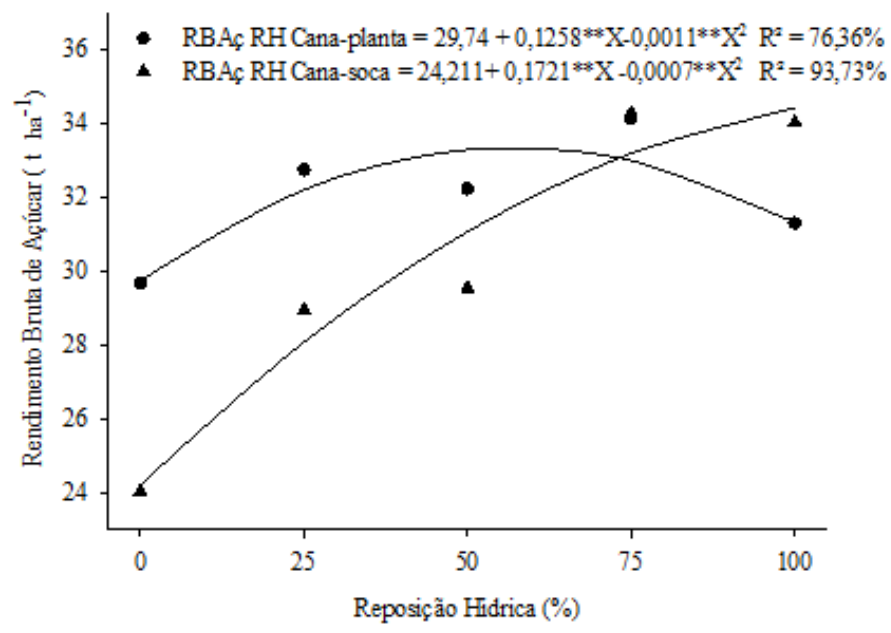

${ }^{* *} \mathrm{e}^{*}$ significativo respectivamente a $(\mathrm{p}<0,01$ e 0,05$)$ segundo teste $\mathrm{F}$.

Figura 7. Rendimento bruto de açúcar em função da Reposição Hídrica x Ciclo na cana-de-açúcar, Rio Verde, Goiás, safras 2011/2012 e 2012/2013.

Para o rendimento bruto de açúcar, o desdobramento do fator $\mathrm{N}$ dentro de $\mathrm{RH}$ houve efeito significativo somente para $100 \%$ de $\mathrm{RH}$, sendo que a maior média foi observada Com $\mathrm{N}$, resultando em um aumento de 22,07\%. Já para o fator $\mathrm{C}$ dentro de $\mathrm{RH}$ a maior média foi observada na Cana-planta, resultando em aumentos de 19,0 e $11,61 \%$ comparado a Canasoca, respectivamente para a $\mathrm{RH} 0$ e $25 \%$ conforme a Tabela 7.

Tabela 7. Teste de media para o desdobramento do fator N x RH e C x RH para Rendimento bruto de açúcar na cana-de-açúcar, Rio Verde, Goiás, safras 2011/2012 e 2012/2013.

\begin{tabular}{cccc}
\hline \multicolumn{4}{c}{ Rendimento bruto de açúcar (RBAç) } \\
\hline $\mathrm{N}$ & \multicolumn{3}{c}{$100 \%$} \\
\hline ComN & \multicolumn{3}{c}{ Médias $\left(\mathrm{t} \mathrm{ha}^{-1}\right)$} \\
SemN & $0 \%, 70 \mathrm{a}$ & $28,60 \mathrm{~b}$ \\
\hline $\mathrm{C}$ & & & $25 \%$ \\
\hline & $29,6662 \mathrm{a}$ & & Médias $\left(\mathrm{t} \mathrm{ha}^{-1}\right)$ \\
Cana-planta & & $32,7375 \mathrm{a}$ \\
Cana-soca & $24,0287 \mathrm{~b}$ & & $28,9362 \mathrm{~b}$ \\
\hline
\end{tabular}

* Médias seguidas de mesma letra nas colunas não diferem entre si segundo teste Tukey a 5\% de probabilidade, FV Fontes de variação; N - nitrogênio; C - ciclo.

\section{CONCLUSÕES}

O nitrogênio influenciou a variável altura de planta e diâmetro de colmos na fase III. Houve interação do nitrogênio com o ciclo na variável altura de planta. O nitrogênio apresentou maior efeito para altura de planta e diâmetro de colmos em condições de canaplanta.

A reposição hídrica apresentou maior efeito para altura de planta em condições de cana-soca. Independentemente da fase fenológica, a cana-de-açúcar variedade RB 855453 se mostrou responsiva a irrigação, aumentou sua produtividade com o aumento da reposição hídrica.

A reposição hídrica promoveu aumento do rendimento bruto de açúcar até 59,34\%, atingindo valores de até 31,60 t ha ${ }^{-1}$. Já a reposição hídrica combinada com a adubação nitrogenada promoveu aumento linear do rendimento bruto de açúcar.

\section{AGRADECIMENTOS}

Os autores agradecem ao Conselho Nacional de Desenvolvimento Científico e 
Tecnológico (CNPq); à Coordenação de Aperfeiçoamento de Pessoal de Nível Superior (CAPES) a Fundação de Amparo à Pesquisa do Estado de Goiás (FAPEG) e ao IF Goiano Campus Rio Verde, pelo apoio financeiro e estrutural.

\section{REFERÊNCIAS BIBLIOGRÁFICAS}

ALLEN, R. G.; PEREIRA, L. S.; RAES, D. Crop evapotranspiration. Rome, 1998. 297 p. (FAO Irrigation and Drenage Paper, 56).

CALDAS, C. Manual de análises selecionadas para indústrias sucroalcooleiras. Maceió: Sindicato da Indústria e do Álcool do Estado de Alagoas, 1998. 424p.

CARR, M. K. V.; KNOX, J. W. The water relations and irrigation requirements of sugarcane (Saccharum officinarum): A review. Experimental Agriculture. v.47 n.1, p.1-25, 2011.

CARVALHO, C. M.; AZEVEDO, H. M.; DANTAS NETO, J.; FARIAS, C. H. A.; SILVA, C. T. S.; GOMES FILHO, R. R. Rendimento de açúcar e álcool da cana-deaçúcar submetida a diferentes níveis de irrigação. Revista Brasileira de Ciências Agrárias, v.4, n.1, p.72-77, 2009.

CINTRA, J. E. V.; FERREIRA, G. H.; BRASIL, R. P. C. Viabilidade da irrigação suplementar na fase inicial de desenvolvimento da cana-de-açúcar (Saccharum ssp.) em regiões com déficit hídrico. Nucleus, Edição Especial, p.111-119, 2008.

CONSECANA. Conselho dos produtores de cana-de-açúcar - CPCA. Açúcar, Álcool do Estado de São Paulo -Consecana -. Manual de instruções. 5.ed. Piracicaba, 2006. 112p.

DALRI, A. B. CRUZ, R. L. Produtividade da cana-de-açúcar fertirrigada com $\mathrm{N}$ e $\mathrm{K}$ via gotejamento subsuperficial. Engenharia Agrícola, v.28, n.3, p.516-524, 2008.
DANTAS NETO, J.; FIGUEIRÊDO, J. L. C.; FARIAS, C. H. A.; AZEVEDO, H. M.; AZEVEDO, C. A. V. Resposta da cana-deaçúcar, primeira soca, a níveis de irrigação e adubação de cobertura. Revista Brasileira de Engenharia Agrícola e Ambiental, v.10, n.2, p.283-288, 2006.

DIOLA, V.; SANTOS, F. Fisiologia. In: SANTOS, F.; BORÉM, A.; CALDAS, C. (Eds.). Cana-de-açúcar: bioenergia, açúcar e etanol - tecnologias e perspectivas. 2 ed. Viçosa: Os Editores, 2012. p. 25-49.

EMBRAPA. Sistema brasileiro de classificação de solos. Empresa brasileira de pesquisa agropecuária. 2.ed. Rio de Janeiro: Ministério da Agricultura e do abastecimento, 2013. 306p.

FARIAS, C. H. A.; FERNANDES, P. D.; AZEVEDO, H. M.; DANTAS NETO, J. Índices de crescimento da cana-de-açúcar irrigada e de sequeiro no Estado da Paraíba. Revista Brasileira de Engenharia Agrícola e Ambiental, v.12, n.4, p.356-362, 2008.

FERREIRA, D. F. Sisvar: a computer statistical analysis system. Ciência e Agrotecnologia, v.35, n.6, p.1039-1042, 2011.

FRANCO, H. C. J.; OTTO, R.; FARONI, C. E.; VITTI, A. C.; OLIVEIRA, E. C. A.; TRIVELIN, P. C. O. Nitrogen in sugarcane derived from fertilizer in Brazilian field conditions. Field Crops Research, v.121, n.1, p.29-41, 2011.

GAVA, G. J. C.; SILVA, M. A.; SILVA, R. C.; JERONIMO, E. M.; CRUZ, J. C. S.; KÖLLN, O. T. Produtividade de três cultivares de cana-deaçúcar sob manejos de sequeiro e irrigado por gotejamento. Revista Brasileira de Engenharia Agrícola e Ambiental, v.15, n.3, p.250-255, 2011.

INMAN-BAMBER, N. G.; SMITH, D. M. Water relations in sugarcane and response to water deficits. Field Crops Research, v.92, n.2-3, p.185-202, 2005. 
MOURA, M. V. P. S.; FARIAS, C. H. A.; AZEVEDO, C. A. V.; DANTAS NETO, J.; AZEVEDO, H. M.; PORDEUS, R. V. Doses de adubação nitrogenada e potássica em cobertura na cultura da cana-de -açúcar, primeira soca, com e sem irrigação. Ciência Agrotécnica, v.29, n.4, p.753-760, 2005.

OLIVEIRA, E. C. A; GAVA, G. J. C.; TRIVELIN, P. C. O.; OTTO, R.; FRANCO, H. C. J. Determining a critical nitrogen dilution curve for sugarcane. Journal of Plant Nutrition and Soil Science, v.176, n.5, p.712723, 2013.

ROSSETTO, R. Maturação da cana-de-açúcar. $<$ http://www.agencia.cnptia.embrapa.br /gestor/cana-deacucar/arvore/CONTAG01_90_221220061548 41.html>. 10 Out. 2013.

SEGATO, S. V.; PINTO, A. S.; NÓBREGA, J. C. M. Atualização em produção de cana-deaçúcar, $1^{\mathrm{a}}$ Ed., Piracicaba, 415 p., 2006.

SINGH, P. N.; SHUKLA, S. K.; BHATNAGAR, V. K. Optimizing soil moisture regime to increase water use efficiency of sugarcane (Saccharum spp. Hybrid complex) in subtropical India. Agricultural Water Management, v.90, n.12, p.95-100, 2007.

SMIT, M. A.; SINGELS, A. The response of surgarcane canopy development to water stress. Field Crops Research, v.98, n.2-3, p.91-97, 2006.

SOUSA, D. M. G.; LOBATO, E. (Eds). Cerrado: correção do solo e adubação. 2. ed. Brasília: Embrapa Informação Tecnológica/Embrapa-CPA, 2004. $416 \mathrm{p}$.

THORNTHWAITE, C. W.; MATHER, J. R. The water balance. Centerton: Drexel Institute of Technology, Laboratory of Climatology, 1955. 104 p. (Publications in Climatology, v. 8, n. 1).

WIEDENFELD. B.; ENCISO, J. Sugarcane responses to irrigation and nitrogen in semiarid South Texas. Agronomy Journal, v.100, n.3 , p.665-671. 2008. 